\title{
Modernisation of High Power Laboratory to fulfill the technical and qualitative conditions for tests according to standards in force
}

\author{
Dr. Eng. George CURCANU, Eng. Corneliu CHICIU, Eng. Constantin ILINCA, Eng. Horia IONESCU \\ R\&D National Institute ICMET-Craiova, 118A Decebal Blvd, 200746 Craiova, Romania \\ phone: +40351 402427; +40351404 888; +40351404889 \\ fax: +40351404890; +40251415482 \\ http://www.icmet.ro,email: LMP@icmet.ro
}

KEYWORDS: electromagnetic fields, tests, standards, data acquisition and processing

\begin{abstract}
High Power Laboratory was modernised by using new electrical apparatus and equipment in order to limit the perturbations due to environment and electromagnetic fields for qualitative improvement of tests performed at high currents and high voltages, according to standards in force. There are also presented the methods for limiting these effects within electric tests laboratories.
\end{abstract}

\section{Introduction}

High Power Laboratory is designed for type tests of electric apparatus and equipment at high currents according to standards in vigour. Testing diagram uses electric circuits made from motor-generator groups, medium voltage circuit-breakers and switchgears, making switches, motors, power transformers, busbars, capacitors, resistors, measure transducers (shunts, current transformers, voltage dividers). [1,2,4]
Due to very strong stresses at high currents and high voltages, arises the problem of achieving such testing and measuring circuits which must contain environmentfriendly materials and, on the other part, not to be affected by very intense electric and magnetic fields [1]. Thus, at initiation of high short-circuit currents and also at tests accompanied by high electric fields in secondary circuits, are induced currents and voltages which can affect the results of measurements if avoiding measures are not taken.

\section{Particularities regarding testing and measuring circuit}

Testing circuit from High Power Laboratory of ICMET Craiova uses a direct scheme. [2,3,4] As resulting from standards requirements (Figure 1), main testing and measuring circuits were modernised with new apparatus, equipment, measuring and recording devices which avoid negative effects on environment and those of electromagnetic fields on staff. [3,4]

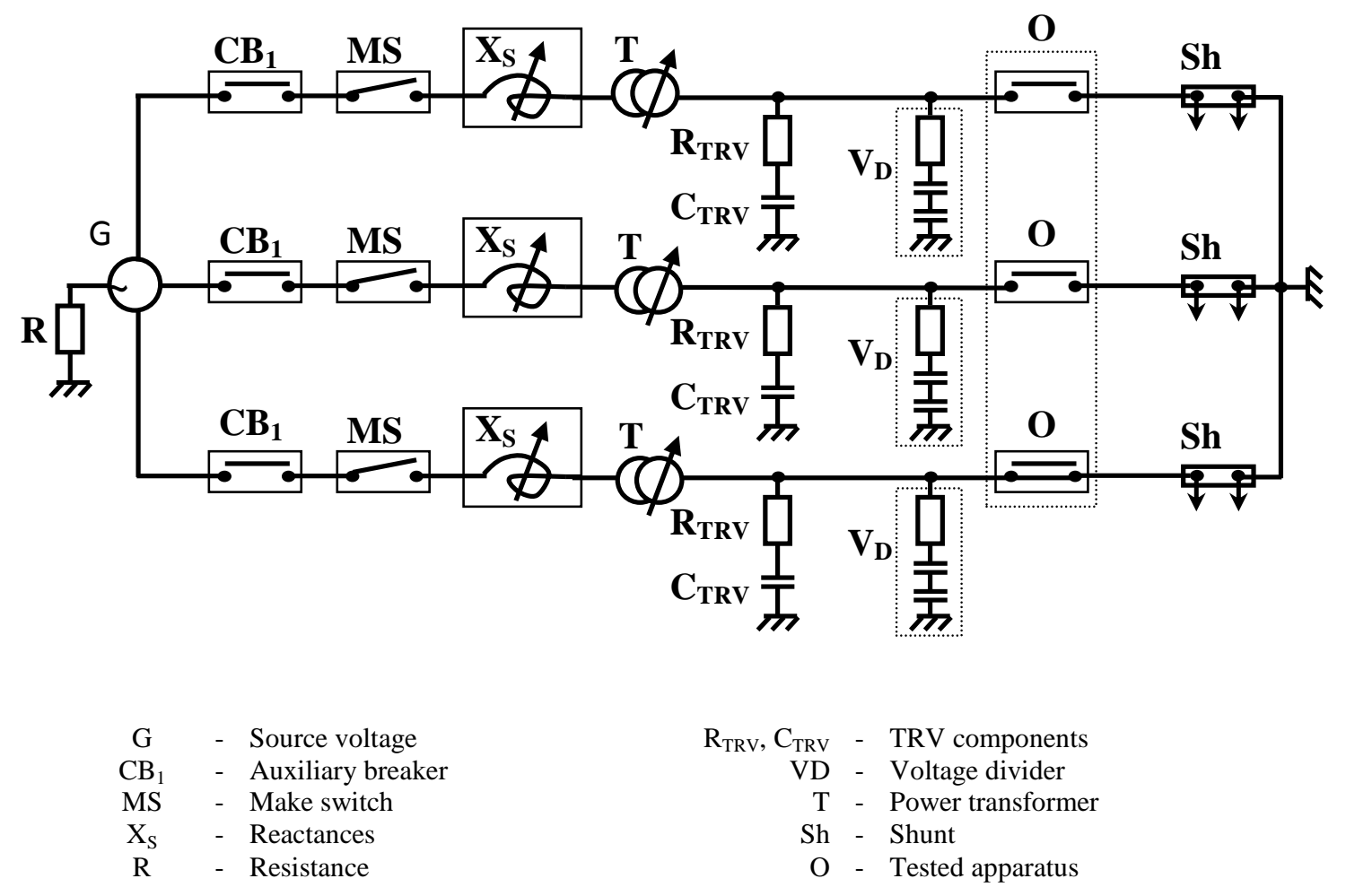

Figure 1 - Test circuit diagram 


\subsection{Testing circuits}

Vacuum circuit-breakers from $6 \mathrm{kV}$ supply voltage (12 kV/1250 A, $12 \mathrm{kV} / 2500 \mathrm{~A}$ ) were chosen to replace minimum-oil circuit-breakers which presented fire risks and pollution by explosion and SF6 circuit-breakers also have negative environment impacts due to gas losses. [3] Vacuum circuit-breakers have not any damaging influence on environment and no explosion risks. Reduced number of components and simplified construction made them more compact and with reduced dimensions which offer them better maintenance and longer life duration (over 25 years).

$0.4 \mathrm{kV}$ supply voltage contains low voltage equipment like: circuit-breakers (400 V/2000 A), circuit-breakers (400 V/100, 200, 300, $400 \mathrm{~A})$, disconnectors (400 V/200, $500 \mathrm{~A})$, low voltage fuses $(400 \mathrm{~V} / 50 \div 500 \mathrm{~A})$.

Medium voltage busbars system is passed by short-circuit currents up to $100 \mathrm{kA}$ for $1-3 \mathrm{~s}$ duration. In order to reduce the strong electrodynamic stresses due to shortcircuit currents was realised a special construction using screened aluminium busbars.

Reactance coils are of cylinder modular type $(0.1 \Omega /$ $12 \mathrm{kV} / 10 \mathrm{kA}$ ) and are realised from copper bands with special resin insulation. Their horizontal placement with frontal output diminishes the effect of mutual inductances and offers a good stability at electrodynamic stresses. $[2,4]$. The capacitors $(6.4 \mu \mathrm{F} / 12 \mathrm{kV})$ from load circuit are realised with modern technologies with ecologic dielectric replacing the old ones with oil dielectric which creates fire risk through explosion. [3]

For measuring test voltages and transient recovery voltages there are used capacitive dividers: $120 \mathrm{kV} / 60 \mathrm{~V}$, $0 \div 3 \mathrm{MHz}$ bandwidth, waterproof varnished fiber glass cylinder.

\subsection{Measuring circuits}

The distortion of useful signal through interference with several perturbations occurs either at measuring transducer, either at connection cable between measuring transducer and recording system. Perturbations can occur due to electromagnetic fields penetration within measuring circuits, formation of closed current loop, multiple reflections, parasite capacitances, intrinsic inductances, too long measuring chain etc. $[1,2,4]$

As high currents transducers there are used compensated coaxial cage shunts $(2 \mathrm{kA} / 2 \mathrm{~V}-180 \mathrm{kA} / 1.8 \mathrm{~V})$. For rapid variable (like making-breaking currents) are used compensated non-inductive shunts. For circuits with no earthing point are used Rogowski coils $(2 \mathrm{kA}-180 \mathrm{kA}$, $0.3 \div 10 \mathrm{MHz}$ bandwidth).

Other perturbation source is given by closed loop through earthing points. For a testing laboratory is not permitted to have two different grounding points which generate leak currents due to potential differences between these different points and due to voltages induced by rapid variable magnetic fields. So measuring devices (shunts, dividers, recording devices) are connected to same earthing point.

Another perturbation source is given by multiple reflections which appears on long connection cables between transducers and recording devices.
Also for external measurements (test cells) appear influences from local radio and TV emissions. In this case are performing screenings and adaptation using adapting impedances.

When TRV is measuring, perturbations appear from intrinsic inductances and parasite capacitances.

To avoid them there are used capacitive dividers with adapting resistances. Also perturbations can appear from too long measuring cables. In this case there are used coaxial double screened (screen earthed) cables. Some cases it is used differential measure schemes. [1]

Measuring circuits connected to recording devices (transient-recorders) are placed perpendicular to high current path and situated in a screened control room. The supply of transient-recorder is performed through galvanic insulated transformer or screened transformer.

These particular aspects were shown related to modernise of measuring circuits using fiber optic connections and transient-recorders with many channels for automatic data recording and processing (Figure 2). [3]

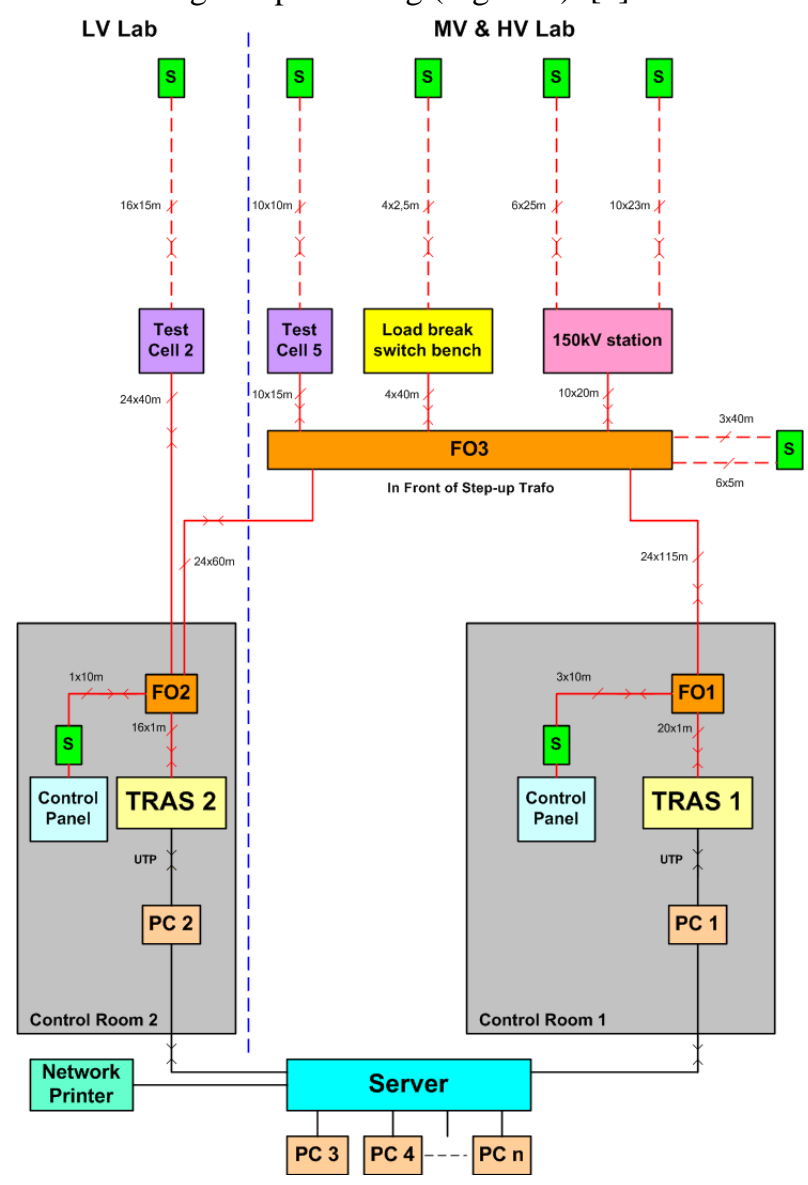

TRAS1,2 - Transient-recorders

FO1,2,3 - fiber optic concentrator and switches (patchfield)

$\mathrm{S}$ - remote module (digitizer)

PC1 ... PCn - local computers

LV Lab - low voltage laboratory

MV Lab - medium voltage laboratory

HV Lab - high voltage laboratory

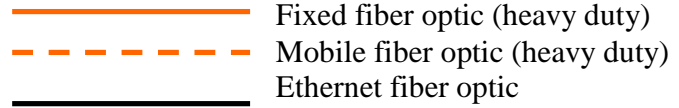

Figure 2 - High Power Laboratory Measuring System 


\subsection{Particularities regarding professional staff}

Laboratory staff are entering testing and measuring circuits only within test responsible witness. There are acoustic and optic warning systems during the tests. Staff is protected during tests within control rooms and is not exposed to electromagnetic fields or to possible explosions of tested apparatus.

However operative witness staff can feel the effects of electromagnetic fields.

In this aspect were established maximum values of exposure at short time electromagnetic fields. [1,5,6]

Communication between control room, machines room and circuit preparing staff is performed through digital wireless interphones.

\section{Experiments for verification of internal arc test on current transformer}

In order to have the guarantee of safety operation in exploitation, the current transformer is submitted to internal arc test according to the international standard IEC 61869-1/2007.

Test parameters for $145 \mathrm{kV}, 1000-2000 / 5 / 5 / 5 / 5$ A current transformer were the following:

- applied voltage: $35.7 \mathrm{kV}$

- $\quad$ short-circuit current: $20 \mathrm{kA}$

- (peak) value of short-circuit current: $54 \mathrm{kA}$

- duration of short-circuit: $0.2 \mathrm{~s}$

Test circuit is single phase. Test arrangement is presented in Figure 3 [7].

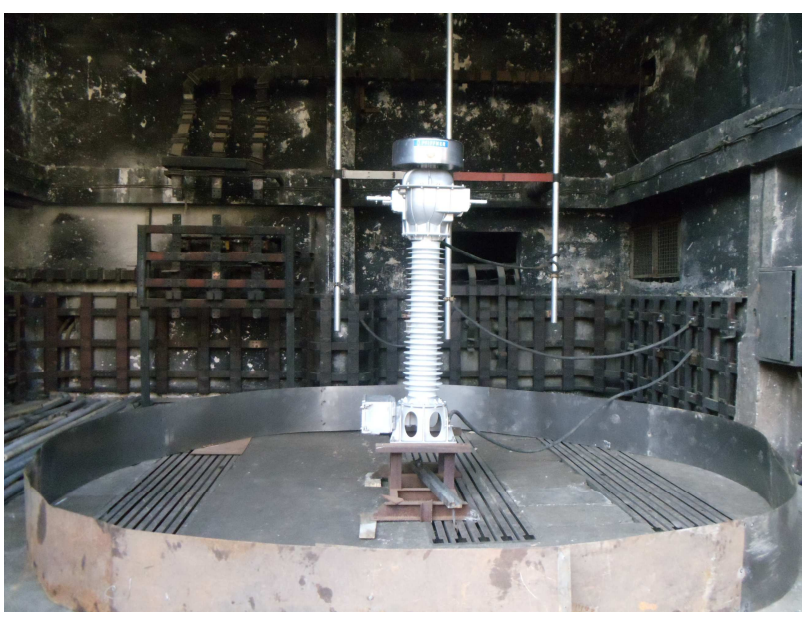

Figure 3 - The current transformer in the test circuit

Measuring circuit is similar to circuit presented in Figure 2. Data acquisition system is an transient-recorder characterised by parameters: number of channels: 24; sampling rate: $100 \mathrm{MS} / \mathrm{s}$; on-board memory: $1 \mathrm{~GB}$; precision: 16 bit. Using Intranet Server, recorded data and oscillograms are sent to protocol room and test responsible office.

Aspects regarding test evolution were recorder with a color fast speed camera, having maximum resolution $1024 \times 1024$ pixels at frame rate $1000 \mathrm{fps}$ and internal memory $4 \mathrm{~GB}$, from the endowment of laboratory.

Test program is presented below:

- Current calibration test at $I_{p}=54 \mathrm{kA}, I_{k}=20 \mathrm{kA}$, $\mathrm{t}_{\mathrm{k}}=0.2 \mathrm{~s}$.

- Voltage calibration test at $U_{a p}=35.7 \mathrm{kV}, \mathrm{t}_{\mathrm{k}}=0.2 \mathrm{~s}$

- Internal arc test at: $U a p=35.7 \mathrm{kV}, \mathrm{I}_{\mathrm{p}}=54 \mathrm{kA}$, $\mathrm{I}_{\mathrm{k}}=20 \mathrm{kA}, \mathrm{t}_{\mathrm{k}}=0.2 \mathrm{~s}, \mathrm{f}=50 \mathrm{~Hz}$.

Test results are presented in Table 1 by oscillogram processing (Figure 4) [7].

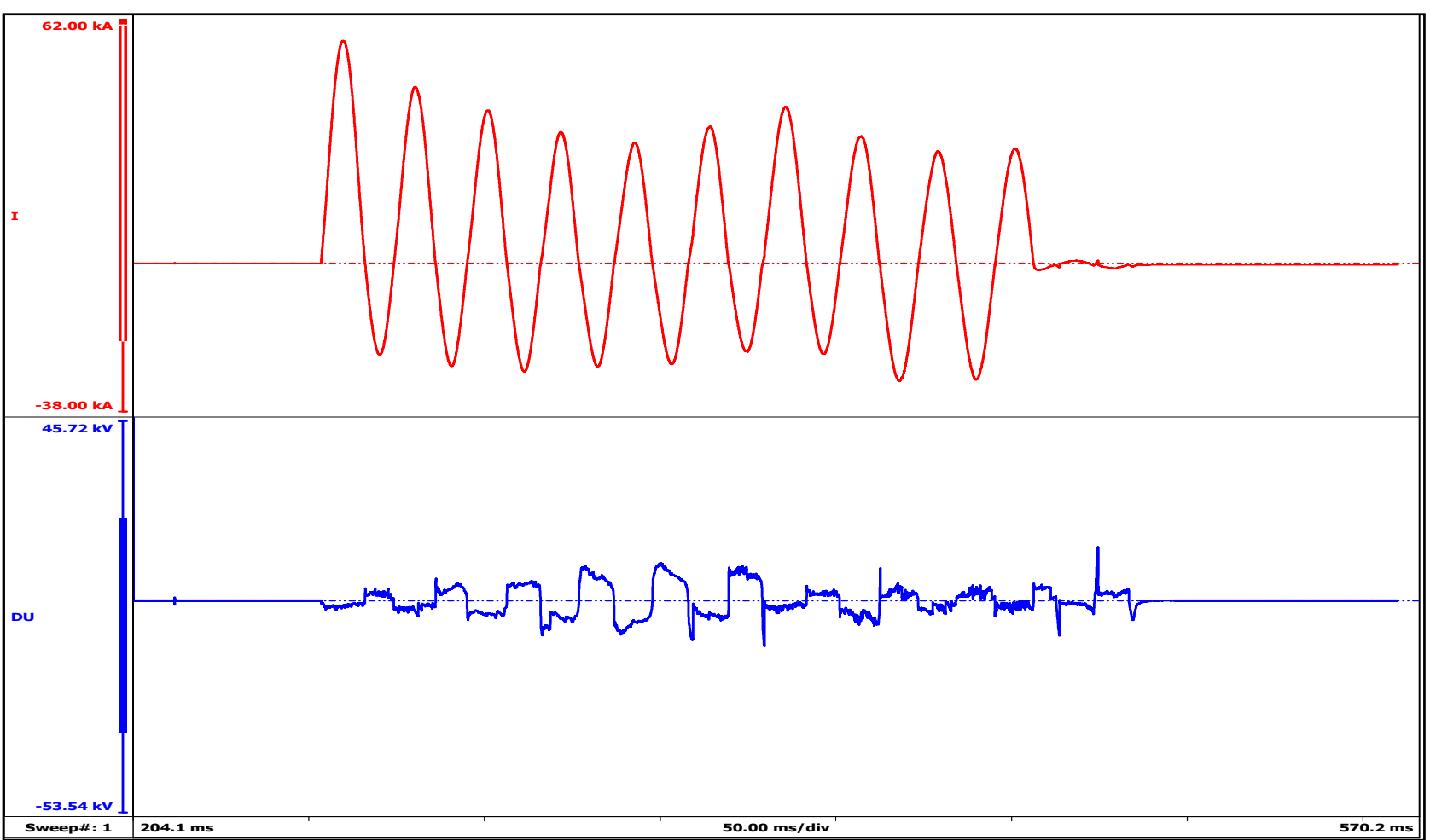

Figure 4 - Oscillogram from internal arc test 
Table 1 - Results obtained at tests

\begin{tabular}{|c|c|c|c|c|c|}
\hline $\begin{array}{c}\mathbf{U}_{\mathbf{a p}} \\
{[\mathbf{k V}]}\end{array}$ & $\begin{array}{c}\mathbf{I}_{\mathbf{p}} \\
{[\mathbf{k A}]}\end{array}$ & $\begin{array}{c}\mathbf{I}_{\mathbf{k}} \\
{[\mathbf{k A}]}\end{array}$ & $\begin{array}{c}\mathbf{t}_{\mathbf{k}} \\
{[\mathbf{s e c} .]}\end{array}$ & $\begin{array}{c}\Delta \mathbf{U} \\
{[\mathbf{k V}]}\end{array}$ & $\mathbf{R e m a r k s}$ \\
\hline- & 56.3 & 20.8 & 0.2 & - & Current calibration \\
\hline 35.7 & - & - & 0.2 & - & Voltage calibration \\
\hline 35.7 & 55.6 & 20.6 & 0.2 & 5.3 & Internal arc test \\
\hline
\end{tabular}

Symbols used in Table 1:

$\mathrm{U}_{\mathrm{ap}}$ - Applied voltage on the one primary winding terminal and core connected to earth

$\mathrm{I}_{\mathrm{p}}$ - Peak values of short-circuit withstand current

$\mathrm{I}_{\mathrm{K}}$ - R.m.s. value of short-circuit withstand current

$t_{k}$ - The duration of short - circuit

$\Delta \mathrm{U}$ - Voltage drop on arc

\section{Conclusions}

By modernisation measures of High Power Laboratory on the basis of European funds [2] there were solved the problems related to screening both testing and measuring circuits. In this aspect the most important it is implementation of a new recording and measuring system by using - in measuring chain - new devices as fiber optic, optical emitters and receivers, transient recorders etc. which ensure both galvanic separation and electromagnetic perturbations immunity (as original contribution).

\section{REFERENCES:}

[1] Titihazan V; Negru V; Crisan S; Curcanu G; Titihazan M - Problems of electromagnetic compatibility in high voltage and high power laboratories - Analele Universitatii Oradea, 2002.

[2] Curcanu G - Testing and diagnosis of electric equipment and apparatus at high currents Editura SITECH, Craiova, 2000.
[3] *** - Modernization of High Power Laboratory (LMP) to reach technical and qualitative level corresponding to European Union requirements - POS CCE - AXA II Project no. 86, 2009.

[4] Chiciu C, Ilinca C, Curcanu G - High Power Laboratory and its role in the development of high voltage apparatus - National Symposium of High Voltage Techniques, Craiova, 2001.

[5] *** - Guide pour l'etablissement de limites d'exposition aux champs electriques, magnetiques, electromagnetiques, champs alternatifs de frecquence variable dans le temps jusqu'à $300 \mathrm{GHz}$ - ICNIRD - International Commission on Non-Ionising Radiation Protection, 2001.

[6] Curcanu G, Ionescu H, Truta D, Chiciu C Aspects regarding modernization of High Power Laboratory for limiting the effects due to electromagnetic fields according to EMC requirements - CNE Proceedings Conferences, Neptun, Romania, 2010.

[7] *** - Test report No. $10988 / 2010$ for $145 \mathrm{kV}$, 1000-2000/5/5/5/5 A Current Transformer. 